\title{
Formation of Non-Abelian Monopoles Connected by Strings
}

\author{
Yifung $\mathrm{Ng}^{1,2}$, T.W.B. Kibble ${ }^{3}$ and Tanmay Vachaspati ${ }^{1,2}$ \\ ${ }^{1}$ Institute for Advanced Study, Princeton, NJ 08540 \\ ${ }^{2}$ CERCA, Department of Physics, Case Western Reserve University, Cleveland, OH 44106-7079 \\ ${ }^{3}$ Blackett Laboratory, Imperial College, London SW7 2AZ, United Kingdom.
}

\begin{abstract}
We study the formation of monopoles and strings in a model where $S U(3)$ is spontaneously broken to $U(2)=[S U(2) \times U(1)] / \mathbb{Z}_{2}$, and then to $U(1)$. The first symmetry breaking generates monopoles with both $S U(2)$ and $U(1)$ charges since the vacuum manifold is $\mathbb{C} P^{2}$. To study the formation of these monopoles, we explicitly describe an algorithm to detect topologically non-trivial mappings on $\mathbb{C} P^{2}$. The second symmetry breaking creates $\mathbb{Z}_{2}$ strings linking either monopole-monopole pairs or monopole-antimonopole pairs. When the strings pull the monopoles together they may create stable monopoles of charge 2 or else annihilate. We determine the length distribution of strings and the fraction of monopoles that will survive after the second symmetry breaking. Possible implications for topological defects produced from the spontaneous breaking of even larger symmetry groups, as in Grand Unified models, are discussed.
\end{abstract}

Topological defects are formed in a vast array of laboratory systems and may also have formed during a cosmological phase transition [1]. The statistical properties at formation of the simplest of defects have been studied quite extensively in the context of cosmology [2] and more recently in a variety of different condensedmatter systems. Experiments have been performed to observe the spontaneous formation of defects in nematic liquid crystals [3, 4, 5], in superfluid ${ }^{3} \mathrm{He}$ [6, 7] and in superconductors [8, 9]. In most particle physics applications, the vacuum manifold can be quite complex, and hybrid topological defects may be formed. These may consist of monopoles connected by strings or walls that are bounded by strings (see for example [10]).

In this paper we study the formation of non-Abelian monopoles that subsequently get connected by strings due to a second non-Abelian symmetry breaking. More specifically, we study monopoles formed in the symmetry breaking

$$
S U(3) \rightarrow U(2) \equiv[S U(2) \times U(1)] / \mathbb{Z}_{2} .
$$

The fundamental monopoles carry both $S U(2)$ and $U(1)$ charge and may be labeled by a pair of charges, $(1, \pm 1)$, where the first entry (with no sign) is the $S U(2)$ charge, and the second entry is the $U(1)$ charge. After the monopoles are formed, we consider the further symmetry breaking

$$
S U(2) \rightarrow \mathbb{Z}_{2}
$$

Now all the monopoles will get connected by strings. However, the $S U(2)$ charge is a $\mathbb{Z}_{2}$ charge, and so there are two types of monopole states connected by strings (Fig. 11). The first of these is a monopole-antimonopole bound state i.e. a bound state of $(1,+1)$ and $(1,-1)$. The confining strings will then eventually bring the monopole and antimonopole together and lead to their annihilation. The second possibility is that the string confines a monopole to a monopole i.e. two $(1,+1)$ or two $(1,-1)$ objects. In this case, the confining string will bring together the two monopoles to form a charge 2 object,
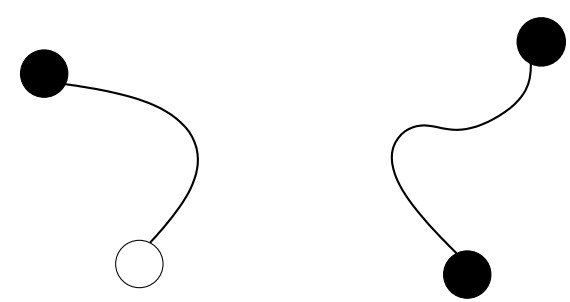

FIG. 1: Two types of confined monopoles in the $S U(3)$ model. The picture on the left represents a monopole and an antimonopole connected by a string. The picture on the right shows two monopoles with the same $U(1)$ charge connected by a string.

$(0, \pm 2)$, that carries no net $S U(2)$ charge but carries twice the basic $U(1)$ charge. One of our aims is to determine the relative number densities of the two types of objects subsequent to the second symmetry breaking stage.

In the context of Grand Unification Theories (GUTs), fundamental magnetic monopoles also carry non-Abelian charges. For example, in the minimal GUT model with $S U(5)$ symmetry, the fundamental monopoles carry $S U(3)$ color, $S U(2)$ weak, and $U(1)$ hypercharge quantum numbers. The formation of magnetic monopoles in the grand unified context occurs due to the non-trivial topology of a very large vacuum manifold and our toy $S U$ (3) model may be expected to capture some of the complications.

One motivation for considering the formation of strings that connect non-Abelian monopoles is that the physics of confinement is not fully understood, and it is possible that non-Abelian magnetic fields also get confined due to quantum or plasma effects [11, 12]. A second related motivation comes from the Langacker-Pi proposal to solve the cosmic monopole over-abundance problem [13]. The scenario assumes that electromagnetic gauge symmetry is spontaneously broken for a period in the early universe. As a result, magnetic monopoles carrying electromagnetic flux will get confined by strings and annihilate effectively. Later the electromagnetic symmetry is restored 
to be consistent with present observations. The breaking of $S U(2)$ in our toy model performs a similar function for this non-Abelian model as does the Langacker-Pi mechanism for the Abelian case, although it does not involve symmetry restoration at low energy. Monopoles again get connected by strings but here they can either annihilate or form charge 2 states. The corresponding scenario in GUTs is more complicated since the monopoles get connected by several different kinds of strings [11, 12], as we discuss in Sec. IV]

We start in Sec. I by describing the field theoretic model under consideration, focussing on the topological aspects. In Sec. II we describe our numerical implementation to study defect formation in the model and the results in Sec. III. We conclude in Sec. IV] by discussing defect formation in an $S U(5)$ GUT model.

\section{MODEL}

Our model contains an $S U(3)$ adjoint field, $\Phi$, whose vacuum expectation value (VEV) implements the symmetry breaking in Eq. (1). Two more $S U(3)$ adjoint fields, $\Psi_{1}$ and $\Psi_{2}$, acquire VEVs to break the $S U(2)$ subgroup of $U(2)$ to $\mathbb{Z}_{2}$ as in Eq. (2). The Lagrangian for the model is

$$
\begin{aligned}
L= & \frac{1}{4} \operatorname{tr}\left[\left(D_{\mu} \Phi\right)^{2}\right]+\frac{1}{4} \sum_{i=1}^{2} \operatorname{tr}\left[\left(D_{\mu} \Psi_{i}\right)^{2}\right] \\
& -\frac{1}{8} \operatorname{tr}\left(X_{\mu \nu} X^{\mu \nu}\right)-V\left(\Phi, \Psi_{1}, \Psi_{2}\right),
\end{aligned}
$$

where $D_{\mu} \Phi=\partial_{\mu} \Phi-i g\left[X_{\mu}, \Phi\right], X_{\mu \nu}$ is the field strength for the $S U(3)$ gauge field $X_{\mu}$, and the potential, $V$, is assumed to have a form that is suitable to give the fields the desired VEVs.

The first stage of symmetry breaking is achieved by the $\mathrm{VEV}$

$$
\Phi=\Phi^{(0)} \equiv \eta T^{8} \equiv \frac{\eta}{\sqrt{3}}\left(\begin{array}{ccc}
1 & 0 & 0 \\
0 & 1 & 0 \\
0 & 0 & -2
\end{array}\right)
$$

where $\eta$ is the energy scale at which the first symmetry breaking occurs and will be set to unity since its value has no effect on the topological structures we are considering. (We could also take $\Phi=g \Phi^{(0)} g^{\dagger}$ for any global $g \in S U(3)$.) The vacuum manifold at this stage is

$$
S U(3) / U(2) \cong \mathbb{C} P^{2}
$$

Points on $\mathbb{C} P^{2}$ are labeled by three complex numbers $\left(z_{1}, z_{2}, z_{3}\right)$, identified under a (complex) rescaling

$$
Z^{T} \equiv\left(z_{1}, z_{2}, z_{3}\right) \cong \kappa\left(z_{1}, z_{2}, z_{3}\right), \quad \kappa \in \mathbb{C}, \quad \kappa \neq 0 .
$$

It will be convenient for us to label the points, following [14], by a point on an octant of a two-sphere given by $\bar{\theta}$ and $\bar{\phi}$, and two phases, $\alpha$ and $\beta$ :

$$
Z^{T}=\left(\sin \bar{\theta} \cos \bar{\phi} e^{i \alpha}, \sin \bar{\theta} \sin \bar{\phi} e^{i \beta}, \cos \bar{\theta}\right),
$$

with $0 \leq \bar{\theta}, \bar{\phi} \leq \pi / 2$ and $0 \leq \alpha, \beta \leq 2 \pi$.

The relation between the field $\bar{\Phi}$ and a point on $\mathbb{C} P^{2}$ is

$$
\Phi=\frac{1}{\sqrt{3}}\left(1-3 \frac{Z Z^{\dagger}}{Z^{\dagger} Z}\right)
$$

The second homotopy group of $\mathbb{C} P^{2}$ is known to be the set of integers $\mathbb{Z}$. A topologically non-trivial configuration can be constructed explicitly by taking $\bar{\phi}=0$. The points on the $\bar{\phi}=0$ sub-manifold are

$$
Z^{T}=\left(\sin \bar{\theta} e^{i \alpha}, 0, \cos \bar{\theta}\right)
$$

and these describe a $\mathbb{C} P^{1}$ subspace of $\mathbb{C} P^{2}$. The points on a two-sphere in physical space, labeled by $(\theta, \phi)$, can be mapped onto this $\mathbb{C} P^{1}$ using

$$
\bar{\theta}=\theta / 2, \quad \bar{\phi}=0, \quad \alpha=\phi, \quad \beta=0 .
$$

Equivalently,

$$
\Phi=\frac{1}{2 \sqrt{3}}\left(\begin{array}{ccc}
3 \cos \theta-1 & 0 & -3 \sin \theta e^{i \phi} \\
0 & 2 & 0 \\
-3 \sin \theta e^{-i \phi} & 0 & -3 \cos \theta-1
\end{array}\right) .
$$

This map represents a simple example of a monopole.

An expression for the topological charge of a monopole can be derived by first constructing the 1-form "gauge potential"

$$
A=\frac{1}{2 i} \frac{Z^{\dagger} d Z-d Z^{\dagger} Z}{Z^{\dagger} Z}
$$

Note that under the "gauge transformation" $Z \rightarrow Z e^{i \lambda}$, which is a special case of (6), $A$ transforms as $A \rightarrow A+d \lambda$. The corresponding field strength 2 -form is

$$
F=d A=\frac{1}{i}\left(\frac{d Z^{\dagger} \wedge d Z}{Z^{\dagger} Z}-\frac{d Z^{\dagger} Z \wedge Z^{\dagger} d Z}{\left(Z^{\dagger} Z\right)^{2}}\right)
$$

Since this 2-form is exact, its integral over a closed twosurface is a topological invariant - and moreover is zero unless the surface contains in its interior a point or points where $Z=0$ (so that $A$ is undefined). So the expression for the topological charge in a volume $V$ with closed boundary $\partial V$ is

$$
Q=\frac{1}{2 \pi} \int_{\partial V} F=\frac{1}{4 \pi} \int_{\partial V} d^{2} S^{i} \epsilon^{i j k} F_{j k} .
$$

There is another way to obtain the expression for the topological charge. We start with the expression known for the 't-Hooft-Polyakov monopole in $S U(2)$ and extend it to $S U(3)$ :

$$
Q=\frac{1}{8 \pi} \int_{\partial V} d^{2} S^{i} f_{a b c} \epsilon^{i j k} n^{a} \partial_{j} n^{b} \partial_{k} n^{c},
$$

where

$$
n^{a}=\frac{Z^{\dagger} T^{a} Z}{Z^{\dagger} Z}
$$


with $a, b, c=1, \ldots, 8$. Here the $T^{a}$ are the generators of $S U(3)$, normalized by $\operatorname{tr}\left(T^{a} T^{b}\right)=2 \delta^{a b}$, the $f_{a b c}$ are structure constants defined by $\left[T^{a}, T^{b}\right]=2 i f_{a b c} T^{c}$, and the integration is over the two sphere at infinity. Also note that the vector $n^{a}$ satisfies $n^{a} n^{a}=4 / 3$. In Appendix $\mathrm{A}$ we show that the two forms for the topolgical charge are equivalent.

It is simple to check that $Q=1$ for the monopole configuration in Eq. (10) and Eq. (11). The formula in Eq. (14) will be useful to locate monopoles in our numerical work described in Sec. II.

The second stage of symmetry breaking is more involved. The fields $\Psi_{j}$ now also acquire VEVs, which are required to lie in the unbroken $S U(2)$ subgroup, and hence commute with $\Phi$. Their magnitudes $\operatorname{tr}\left(\Psi_{j}^{2}\right)$ are fixed by the potential, and they are also required to be mutually orthogonal in the sense that $\operatorname{tr}\left(\Psi_{1} \Psi_{2}\right)=0$. Given a value of $\Phi$ at some spatial point $P$, we need to identify this unbroken subgroup. The standard procedure is to work out commutators of $\Phi$ with $S U(3)$ generators and to find linear combinations of the generators that commute. In practice, it is easier to first rotate $\Phi$, say by an $S U(3)$ rotation $R$, to the reference direction, $\Phi^{(0)}$. We discuss how to choose $R$ below. Then the generators of the unbroken $S U(2)$ sit in the $2 \times 2$ upper left corner while the generator $T^{8}$ of the unbroken $U(1)$ is in the direction of $\Phi^{(0)}$ itself. With respect to $\Phi^{(0)}$, the VEVs of $\Psi_{1}$ and $\Psi_{2}$ can be written in terms of two orthonormal 3 -vectors, $\mathbf{a}$ and $\mathbf{b}$, as $\Psi_{1}^{(0)}=\mathbf{a} \cdot \mathbf{T}$ and $\Psi_{2}^{(0)}=\mathbf{b} \cdot \mathbf{T}$ where

$$
T^{i}=\left(\begin{array}{cc}
\sigma_{i} & \mathbf{0} \\
\mathbf{0} & 0
\end{array}\right), \quad i=1,2,3,
$$

and $\sigma_{i}$ are the Pauli spin matrices. Once $\Psi_{1}^{(0)}$ and $\Psi_{2}^{(0)}$ are constructed, we can rotate all the fields back to the original point using $R^{\dagger}$.

The VEVs of $\Psi_{1}$ and $\Psi_{2}$ break $S U(2)$ down to $\mathbb{Z}_{2}$, which is the center of $S U(2),\left\{\mathbf{1},-\mathbf{1}_{2}\right\}$, i.e. the identity element of $S U(3)$ and $-\mathbf{1}_{2} \equiv \operatorname{diag}(-1,-1,1)$. A string passes through a spatial contour if $\Psi_{1}$ and $\Psi_{2}$ are such that, on going around the contour, these fields are transformed by the element $-\mathbf{1}_{2}$ and not by the identity element. The strings are of the $\mathbb{Z}_{2}$ variety and there is no distinction between a string and an anti-string. Also, there is no known integral formula that can be used to evaluate the winding around the contour.

\section{NUMERICAL IMPLEMENTATION}

To simulate the formation of the monopole-string network, a 3-dimensional cubic lattice is chosen. Each cubic cell is further divided into 24 tetrahedral sub-cells, obtained by connecting the center of the cube to the 8 corners and the centers of the 6 faces (see Fig. 2).

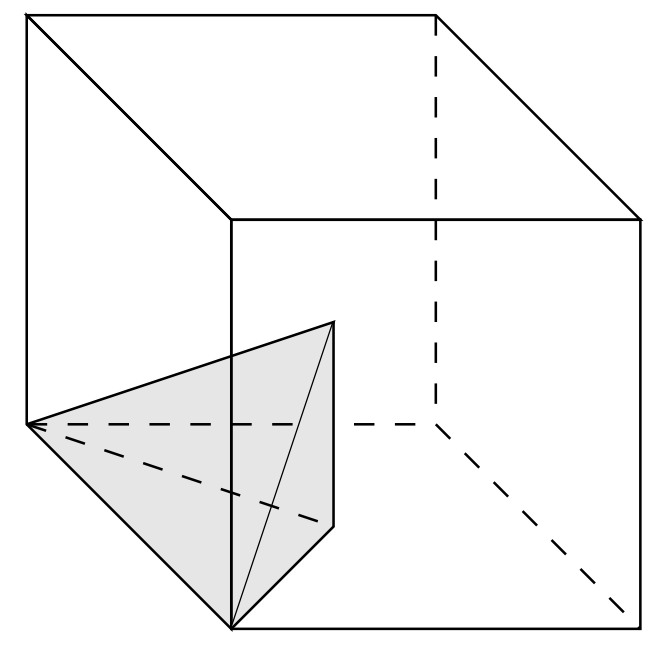

FIG. 2: Each cell of the cubic lattice is sub-divided into 24 tetrahedra. Only one cubic cell and representative tetrahedron are shown.

The next step is to assign random points of $\mathbb{C} P^{2}$ at each point on the lattice, including the centers of the cubic cells and their faces. Now, the unique $S U(3)$ invariant metric on $\mathbb{C} P^{2}$ is the Fubini-Study metric

$$
d s^{2}=\frac{d Z^{\dagger} d Z}{Z^{\dagger} Z}-\frac{d Z^{\dagger} Z Z^{\dagger} d Z}{\left(Z^{\dagger} Z\right)^{2}}
$$

or, in terms of the parameter choice of (77),

$$
\begin{aligned}
d s^{2}= & d \bar{\theta}^{2}+\sin ^{2} \bar{\theta} d \bar{\phi}^{2} \\
& +\sin ^{2} \bar{\theta} \cos ^{2} \bar{\phi}\left(1-\sin ^{2} \bar{\theta} \cos ^{2} \bar{\phi}\right) d \alpha^{2} \\
& -2 \sin ^{4} \bar{\theta} \cos ^{2} \bar{\phi} \sin ^{2} \bar{\phi} d \alpha d \beta \\
& +\sin ^{2} \bar{\theta} \sin ^{2} \bar{\phi}\left(1-\sin ^{2} \bar{\theta} \sin ^{2} \bar{\phi}\right) d \beta^{2} .
\end{aligned}
$$

Hence the $S U(3)$-invariant measure on $\mathbb{C} P^{2}$ is

$$
\sqrt{g} d \bar{\theta} d \bar{\phi} d \alpha d \beta=\sin ^{3} \bar{\theta} \cos \bar{\theta} \sin \bar{\phi} \cos \bar{\phi} d \bar{\theta} d \bar{\phi} d \alpha d \beta .
$$

Thus the assignment is done by drawing $0 \leq \sin ^{4} \bar{\theta} \leq 1$, $0 \leq \sin ^{2} \bar{\phi} \leq 1,0 \leq \alpha \leq 2 \pi$ and $0 \leq \beta \leq 2 \pi$ from uniform distributions, and then constructing $Z$ as in Eq. (77). The four vertices of a spatial tetrahedron then get mapped on to a tetrahedron in $\mathbb{C} P^{2}$ which we will denote by $\left(Z_{1}, Z_{2}, Z_{3}, Z_{4}\right)$. To find out if this tetrahedron in $\mathbb{C} P^{2}$ is topologically non-trivial (i.e. incontractable) we use a discrete version of the charge formula in Eq. (14)

$$
Q=\frac{1}{2 \pi} \sum_{\{i j k\}} \alpha_{\{i j k\}},
$$

where the sum is over the four triangular faces of the tetrahedron (with positive orientation), and for each face,

$$
\alpha_{\{i j k\}}=\arg \left(Z_{i}^{\dagger} Z_{k} Z_{k}^{\dagger} Z_{j} Z_{j}^{\dagger} Z_{i}\right),
$$

where we require $\alpha_{\{i j k\}}$ to lie within the range $[-\pi, \pi]$. We can explicitly check that small changes in the $Z_{i}$ do 
not affect $Q$, thus showing that even the discrete formula is topological.

One can also check that Eq. (21) agrees with Eq. (14). The charge $Q$ is the integral of $F / 2 \pi$ over a large sphere, which can be broken up into the sum of the four separate contributions from the individual faces of the tetrahedron. Each of these can be expressed as the integral of the 1 -form $A / 2 \pi$ around the perimeter. In discretized form, the integral of $A$ along the 1-2 link becomes $\arg \left(Z_{2}^{\dagger} Z_{1}\right)$ (see Eq. (12) ) and so the magnetic flux through the triangular plaquette $\{123\}$, is found by summing the contributions from the three edges,

$$
\begin{gathered}
\oint \mathbf{d x} \cdot \mathbf{A}=\arg \left(Z_{2}^{\dagger} Z_{1}\right)+\arg \left(Z_{3}^{\dagger} Z_{2}\right)+\arg \left(Z_{1}^{\dagger} Z_{3}\right) \\
+2 \pi n,
\end{gathered}
$$

where $n$ is an integer and the extra term, $2 \pi n$, in Eq. (23) is included because each of the phases is ambiguous up to $\pm 2 \pi$. This can also be seen as a gauge ambiguity: a gauge transformation may change the value of $n$. It has a geometric interpretation as well. For the special case of triangles on a $\mathbb{C} P^{1}$ subspace of $\mathbb{C} P^{2}$ (isometric to a sphere of radius $1 / 2$ ), we have shown that the flux through a triangle, found using Eq. (14), is equal to twice the area of the triangle. Thus the ambiguity in the flux in Eq. 23 is equivalent to the ambiguity in choosing between the two complementary spherical triangles with this boundary. We choose the one with the smaller area, so that

$$
\oint \mathbf{d x} \cdot \mathbf{A}=\alpha_{\{123\}} .
$$

Thus Eq. (21) is the discretized version of Eq. (14).

We conjecture that for a general triangle in $\mathbb{C} P^{2}$, not lying on a $\mathbb{C} P^{1}$ subspace, the flux through it may still be equal to twice the area of the minimal surface with that boundary. Choosing the minimal area may be seen as a generalization to areas of the "geodesic rule" for lengths [2]. The rule in general is to choose the minimal value of the integral in Eq. (23).

Next we turn to the formation of strings that connect the monopoles. For this we need to consider a triangular face of a tetrahedron and determine if a string passes through it.

Each vertex of a triangular plaquette has already been assigned a point on $\mathbb{C} P^{2}$, equivalently a $\mathrm{VEV}$ of $\Phi$. It is convenient to label the subgroup that leaves $\Phi_{i}$ invariant as $S U(2)_{i} \times U(1)_{i} / \mathbb{Z}_{2}$. Now we also assign VEVs of $\Psi_{1}$ and $\Psi_{2}$, making sure that these lie in the unbroken $S U(2)$ sector of $S U(3)$ at $Z_{i}$, namely $S U(2)_{i}$, and that they are orthogonal: $\operatorname{tr}\left(\Psi_{1} \Psi_{2}\right)=0$. The precise scheme is as follows.

- The scheme is based on the construction, for each pair of points on $\mathbb{C} P^{2}$, say $Z_{i}$ and $Z_{j}$, of an $S U(3)$ transformation, $R_{j i}$, that transforms $Z_{i}$ to some representative of the point $Z_{j}$ and moreover does so along a geodesic in $\mathbb{C} P^{2}$, i.e. $R_{j i} Z_{i} \cong Z_{j}$. In fact the left-hand side is equal $Z_{j}$ times the phase factor that makes the scalar product with $Z_{i}^{\dagger}$ real (see Appendix (B). In other words, we find

$$
R_{j i} Z_{i}=Z_{j} \frac{Z_{j}^{\dagger} Z_{i}}{\left|Z_{j}^{\dagger} Z_{i}\right|} .
$$

The geodesic condition will be achieved if $R_{j i}$ can be written as

$$
R_{j i}=\exp (i M s)
$$

where $M$ is a suitably chosen normalized combination of the generators $T^{a}$ and $s$ is the geodesic distance between $Z_{i}$ and $Z_{j}$, given by

$$
s=\cos ^{-1}\left(\sqrt{\frac{\left(Z_{i}^{\dagger} Z_{j}\right)\left(Z_{j}^{\dagger} Z_{i}\right)}{\left(Z_{i}^{\dagger} Z_{i}\right)\left(Z_{j}^{\dagger} Z_{j}\right)}}\right) .
$$

A more explicit construction of $R_{j i}$ is described in Appendix B.

Similarly, for each $Z_{i}$, we define an $S U(3)$ transformation $R_{i 0}$ such that $Z_{i}=R_{i 0} Z_{0}$, where $Z_{0}$ is the reference point $(0,0,1)$. (With our choice of representative in (7), no phase factor is needed here.) The matrix $R$ described in the previous section, above Eq. (17), will be one of the $R_{i 0}^{\dagger}$.

- To each vertex of the triangular face is associated a point on $\mathbb{C} P^{2}$ (say $Z_{i}$ ) and two uniformly distributed orthonormal 3 -vectors, $\mathbf{a}_{i}$ and $\mathbf{b}_{i}$ where $i$ labels the vertex of the triangle (see Fig. 3). If we wish, we can construct $\Phi_{i}$ from $Z_{i}$ using Eq. (8). The two remaining fields $\Psi_{1,2}$ may be found from $\mathbf{a}$ and $\mathbf{b}$. We first define

$$
A_{i 0}=\mathbf{a} \cdot \mathbf{T}, \quad B_{i 0}=\mathbf{b} \cdot \mathbf{T},
$$

which are $S U(3)$ matrices lying in the $S U(2)_{0}$ subgroup, with generators $\mathbf{T}$ given by Eq. (17). Then the fields are given by $\Psi_{1}=\eta_{1} A$ and $\Psi_{2}=\eta_{2} B$, where $\eta_{1,2}$ are the magnitudes of these fields, and the normalized $S U(3)$ matrices $A$ and $B$ may be found by using the transformation $R_{i 0}$ :

$$
A_{i}=R_{i 0} A_{i 0} R_{i 0}^{\dagger}, \quad B_{i}=R_{i 0} B_{i 0} R_{i 0}^{\dagger} .
$$

Note that by construction $A_{i}$ and $B_{i}$ belong to $S U(2)_{i}$ and hence commute with $\Phi_{i}$.

- Now we want to compare the symmetry-breaking fields at neighboring vertices. To do this we transport them using the geodesic transformations $R_{j i}$. Transforming $A_{i}$ and $B_{i}$ by parallel transport along a geodesic from $Z_{i}$ to $Z_{j}$, we obtain

$$
A_{j i}=R_{j i} A_{i} R_{j i}^{\dagger}, \quad B_{j i}=R_{j i} B_{i} R_{j i}^{\dagger} .
$$


Next we compare these transported matrices with the corresponding matrices $A_{j}, B_{j}$ defined at the vertex $Z_{j}$. We seek a transformation $S_{j i} \in S U(2)_{j}$ such that

$$
A_{j}=S_{j i} A_{j i} S_{j i}^{\dagger}, \quad B_{j}=S_{j i} B_{j i} S_{j i}^{\dagger} .
$$

In Appendix C we describe our construction of $S_{j i}$ in detail.

- The net rotation of the pair $A_{i}, B_{i}$ as we circumnavigate the triangular face from $Z_{i}$ to $Z_{j}$ to $Z_{k}$ and back to $Z_{i}$ is

$$
S_{\{i j k\}} \equiv S_{i k} R_{i k} S_{k j} R_{k j} S_{j i} R_{j i} .
$$

Note that since this combined transformation leaves invariant all the fields $\Phi_{i}, A_{i}, B_{i}$, it must belong to the unbroken $U(1)_{i}$.

- To determine whether or not a string passes through the $\{i j k\}$ face, we have to compare $S_{\{i j k\}}$ with the transformation $R_{i k} R_{k j} R_{j i}$ without the intervening $S$ factors. Since this transformation leaves $\Phi_{i}$ invariant, it belongs to $S U(2)_{i} \times U(1)_{i} / \mathbb{Z}_{2}$. Moreover, in view of Eq. (22), we know that

$$
R_{i k} R_{k j} R_{j i} Z_{i}=Z_{i} e^{i \alpha_{\{i j k\}}} .
$$

Consequently, we know that the $U(1)_{i}$ factor in this product must be

$$
\exp \left(-\frac{1}{2} i \alpha_{\{i j k\}} \sqrt{3} T_{i}^{8}\right) .
$$

Now let us return to $S_{\{i j k\}}$. Since for example the transformation $S_{j i} \in S U(2)_{j}$ leaves $Z_{j}$ unaltered, it is clear that, regardless of the choice of the $S$ factors, the effect of $S_{\{i j k\}}$ on $Z_{i}$ must be exactly the same as that of the product in Eq. (33). Consequently, the combination

$$
W_{\{i j k\}}=S_{\{i j k\}} \exp \left(\frac{1}{2} i \alpha_{\{i j k\}} \sqrt{3} T_{i}^{8}\right)
$$

must leave $Z_{i}$ invariant, and also not contribute a phase to $Z_{i}$, and hence it belongs to $S U(2)_{i}$. But we know that $W_{\{i j k\}}$ also belongs to $U(1)_{i}$, since it consists of two factors each of which is an element of $U(1)_{i}$. So $W_{\{i j k\}}$ must in fact be one of the two central elements that are common to both $S U(2)_{i}$ and $U(1)_{i}$. If $W_{\{i j k\}}=\mathbf{1}$, the winding is trivial and there is no string through the triangular face. If, however, $W_{\{i j k\}}=-\mathbf{1}_{2}$, then there is a string through the triangular plaquette.

It can be shown (see Appendix D) that if the monopole charge (21) within the tetrahedron is non-zero, then there must be an odd number of faces with strings passing through, while if it is zero there must be an even number. This follows from the fact that each edge, say $(i j)$ appears, with opposite orientation in two faces, and the relevant factors in say $S_{\{i j k\}}$ and $S_{\{j i l\}}$ are inverses of each other: $\left(S_{j i} R_{j i}\right)^{\dagger}=S_{i j} R_{i j}$.

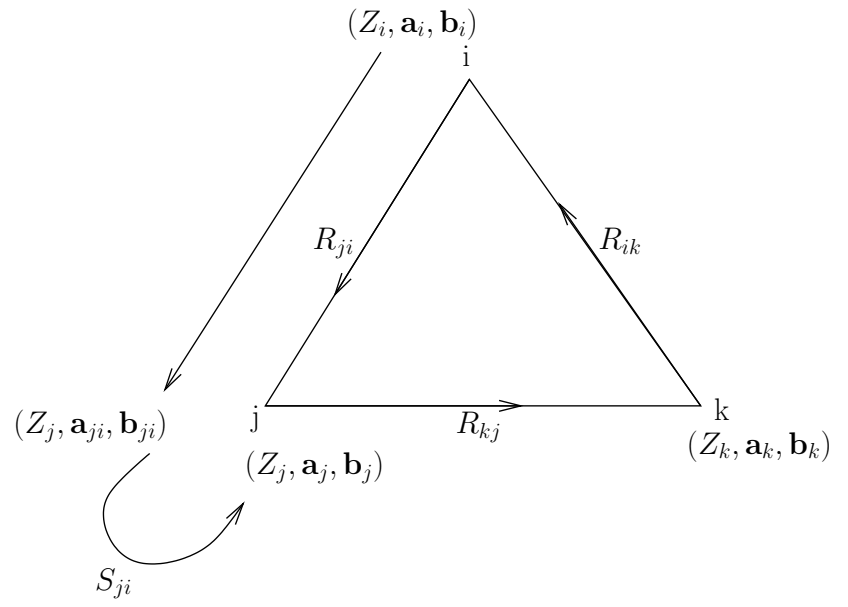

FIG. 3: The algorithm to find strings requires parallel transport of the variables at vertex $i$ along a geodesic on $\mathbb{C} P^{2}$ to the vertex $j$. Then the transported variables are rotated to the assigned variables at $j$, by using an $S U(2)$ geodesic transformation.

To get a better physical sense for this algorithm, it is useful to consider monopole and string formation in the simpler symmetry breaking pattern

$$
S U(2) \rightarrow U(1) \rightarrow 1 .
$$

This example is discussed in Appendix E We should also add that the natural language for our discussion is in terms of fiber bundles since what we have in our model is an $S^{3} / \mathbb{Z}_{2}$ fiber over a $\mathbb{C} P^{2}$ base manifold. The topology of the base manifold, $\mathbb{C} P^{2}$, gives rise to monopoles while the topology of the fiber, $S^{3} / \mathbb{Z}_{2}$, gives rise to strings that may end on monopoles.

\section{RESULTS}

The simulations were done on a cubic lattice of side 12 i.e. in $24 \times 12^{3}$ tetrahedral cells and was repeated 10 times to gain statistics. The probability of having a monopole or antimonopole in a cell is 0.17 . If $N$ is the total number of string segments, then the relative numbers of segments in closed loops, string segments connecting like charge monopoles, and string segments connecting oppositely charged monopoles, are given by

$$
\begin{aligned}
\frac{N_{\text {loops }}}{N} & =0.4 \% . \\
\frac{N_{ \pm \pm}}{N} & =4.2 \% . \\
\frac{N_{+-}}{N} & =95.4 \% .
\end{aligned}
$$

This shows that roughly $4 \%$ of $S U(3)$ monopoles will end up in the doubly charged state and survive annihilation due to strings. 


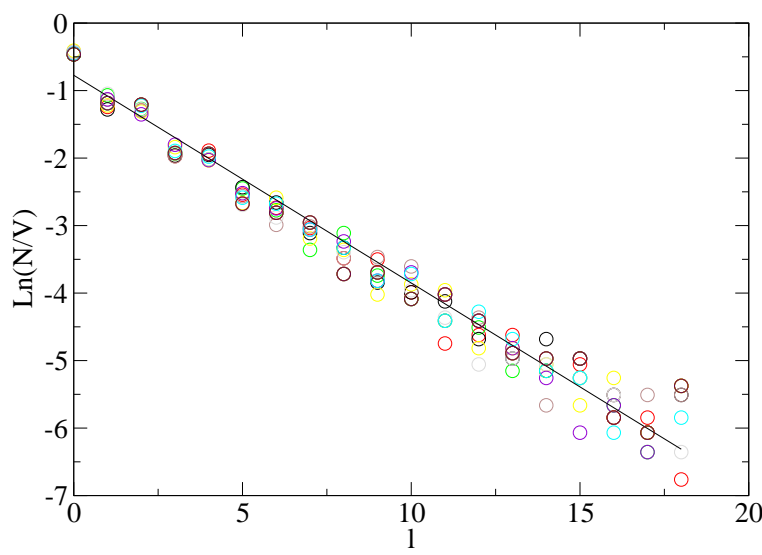

FIG. 4: Logarithm of average number density of strings connecting monopoles and antimonopoles versus string length.

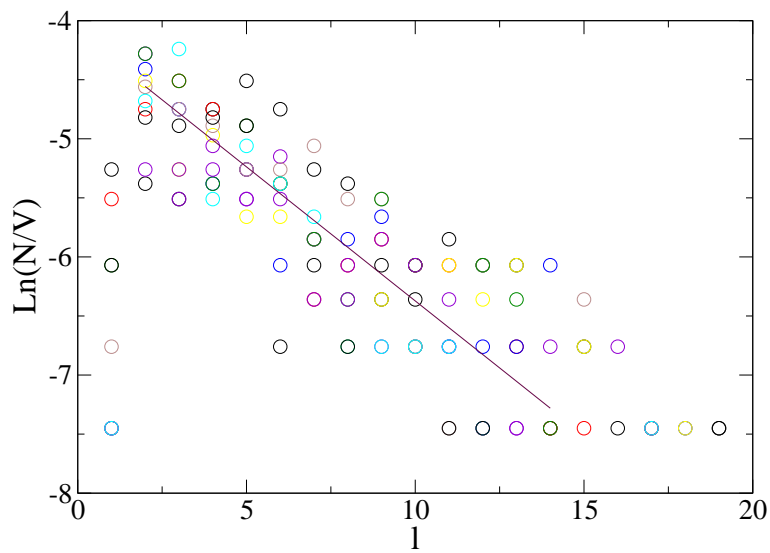

FIG. 5: Logarithm of average number density of monopolemonopole $(++)$ and antimonopole-antimonopole $(--)$ connections versus string length.

The length distribution of +- strings is shown in Fig. 4. Denoting the number density of these strings, i.e. number of segments divided by the volume $\left(12^{3}\right)$, by $n_{+-}$, the least-squares linear fit is

$$
n_{+-}(l)=(0.46 \pm 0.08) e^{-(0.31 \pm 0.03) l}
$$

The corresponding distribution of ++ and -- strings is shown in Fig. 5 and the fit is

$$
n_{ \pm \pm}(l)=(0.02 \pm 0.01) e^{-(0.23 \pm 0.07) l}
$$
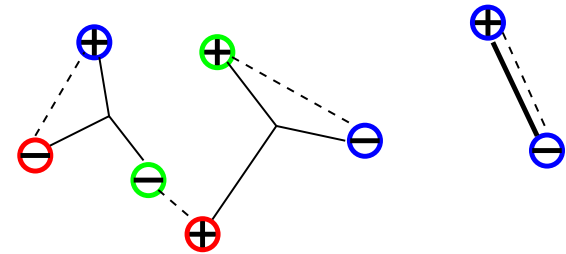

FIG. 6: A cluster of 6 monopoles can form a singlet of $S U(3)$ and $S U(2)$, as in ordinary baryons. A bound state of a monopole and antimonopole is also possible, as in ordinary mesons. The $S U(3)$ charge on a monopole is shown in shades of grey (or in color) and the $S U(2)$ charge as a \pm . We have not shown the $U(1)$ charge. $\mathbb{Z}_{3}$ strings are shown as solid lines; $\mathbb{Z}_{2}$ strings as dashed lines.

\section{DISCUSSION}

We have studied the formation of monopoles connected by strings in an $S U(3)$ model and the results for the distribution of monopoles and strings are summarized in Sec. III. Here we discuss qualitatively how a similar analysis in realistic grand unified models would proceed. Our experience with $S U(3)$ helps us understand and appreciate the difficulties that are likely to be encountered. As an example, consider the minimal grand unified model based on a $S U(5)$ symmetry group. The symmetry breaking pattern is

$$
S U(5) \rightarrow[S U(3) \times S U(2) \times U(1)] / \mathbb{Z}_{3} \times \mathbb{Z}_{2} .
$$

and, if the non-Abelian magnetic charges are confined, the relevant symmetry breakings are

$$
S U(3) \rightarrow \mathbb{Z}_{3}, \quad S U(2) \rightarrow \mathbb{Z}_{2} .
$$

The fundamental magnetic monopoles carry $S U(3)$ and $S U(2)$ charges in addition to the topological $U(1)$ charge. Therefore each monopole will get connected to a $\mathbb{Z}_{3}$ string and another $\mathbb{Z}_{2}$ string. Then isolated clusters of monopoles come in two varieties, similar to known baryons and mesons, as shown in Fig. 6. However, a likely outcome at formation seems to be that, in addition to some isolated baryonic and mesonic clusters, the monopole-string network percolates and we essentially obtain one giant structure, such as depicted in Fig. 7.

It seems hard to explicitly confirm if the network percolates, say by numerical simulation. For example, the vacuum manifold at the first stage of symmetry breaking is 12 dimensional and it also does not fall into a straightforward category like $\mathbb{C} P^{n}$. Determining the distribution of strings is also more complicated since the $S U(3)$ breaking leads to $\mathbb{Z}_{3}$ strings. These problems do not seem insurmountable but are hard enough that we have not attempted to solve them at the present time.

If very few baryonic clusters form and instead an infinite monopole-string network forms, our experience with string networks [15, 16, 17, 18] suggests that the network energy density scales with time and never comes 


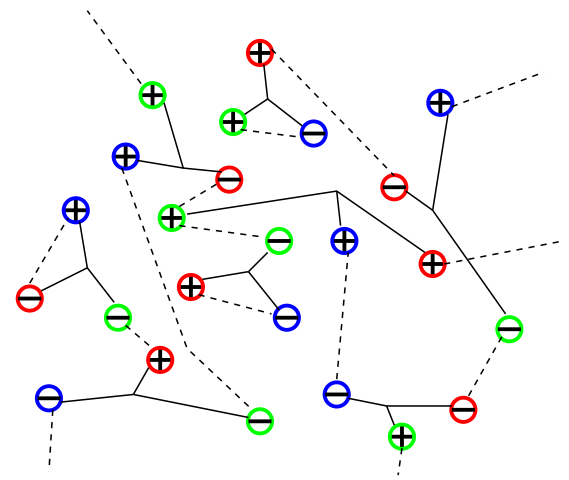

FIG. 7: Drawing of an infinite monopole-string network that could result from $S U(5)$ grand unified symmetry breaking. The three different shades of circles represent the $S U(3)$ color charge and the plus-minus symbols within the circles the $S U(2)$ charge. The $U(1)$ (hypercharge) charge has not been shown. The isolated clusters of monopoles have to occur in $S U(3)$ and $S U(2)$ singlets.

to dominate the universe. Processes such as monopoleantimonopole annihilation and meson formation could dissipate the energy of the network at a rate that is determined by the Hubble expansion. However, this scenario ignores the process of baryon formation from the network. Depending on the rate of this process, we could still have a monopole over-abundance problem coming from the production of baryonic clusters.

\section{Acknowledgments}

We thank Andrew Neitzke and Yuji Tachikawa for very helpful discussions. This work was supported by the U.S. Department of Energy and NASA at Case Western Reserve University.

\section{APPENDIX A: TOPOLOGICAL CHARGE}

We wish to show that the two expressions for the topological charge, Eqs. (14) and (15), are equivalent.

The demonstration follows by using the $S U(3)$ identity

$$
f_{a b c} T_{i j}^{a} T_{k l}^{b} T_{m n}^{c}=2 i\left(\delta_{i n} \delta_{k j} \delta_{m l}-\delta_{i l} \delta_{k n} \delta_{m j}\right) .
$$

where $T^{a}$ are $S U(3)$ generators normalized such that $\operatorname{tr}\left(T^{a} T^{b}\right)=2 \delta^{a b}$, and $f_{a b c}$ are the structure constants defined by $\left[T^{a}, T^{b}\right]=2 i f_{a b c} T^{c}$. The above identity is a generalization of the better known identity for the $S U(2)$ generators $\sigma^{a}$ :

$$
\epsilon_{a b c} \sigma_{i j}^{a} \sigma_{k l}^{b} \sigma_{m n}^{c}=2 i\left(\delta_{i n} \delta_{k j} \delta_{m l}-\delta_{i l} \delta_{k n} \delta_{m j}\right)
$$

Now, if we choose $Z^{\dagger} Z=1$, Eq. (15) can be written

$$
\begin{aligned}
Q= & \frac{1}{8 \pi} f_{a b c} T_{i j}^{a} T_{k l}^{b} T_{m n}^{c} \int d^{2} S^{p} \\
& \epsilon^{p q r}\left(z_{i}^{*} z_{j}\right) \partial_{q}\left(z_{k}^{*} z_{l}\right) \partial_{r}\left(z_{m}^{*} z_{n}\right) .
\end{aligned}
$$

Using (A1) this becomes

$$
\begin{array}{r}
Q=\frac{i}{4 \pi}\left(\delta_{i n} \delta_{k j} \delta_{m l}-\delta_{i l} \delta_{k n} \delta_{m j}\right) \int d^{2} S^{p} \epsilon^{p q r} \\
\left(z_{i}^{*} z_{j}\right)\left(\partial_{q} z_{k}^{*} z_{l}+z_{k}^{*} \partial_{q} z_{l}\right)\left(\partial_{r} z_{k}^{*} z_{m}+z_{m}^{*} \partial_{r} z_{n}\right) .
\end{array}
$$

The contractions lead to factors such as $Z^{\dagger} Z=1$ or else similar factors with derivatives, such as $Z^{\dagger} \partial_{q} Z, \partial_{q} Z^{\dagger} Z$, or $\partial_{q} Z^{\dagger} \partial_{r} Z$. Of the eight terms in (A3), four cancel in pairs, and the other four are equal in pairs, yielding finally

$$
Q=\frac{1}{2 \pi i} \int d^{2} S^{p} \epsilon^{p q r}\left(\partial_{q} Z^{\dagger} \partial_{r} Z-\partial_{q} Z^{\dagger} Z Z^{\dagger} \partial_{r} Z\right),
$$

which, using Eq. (13), is precisely Eq. (14).

\section{APPENDIX B: $S U(3)$ GEODESIC MATRIX}

Here we will construct the $S U(3)$ matrix $R_{j i}$ such that

$$
R_{j i} Z_{i} \cong Z_{j} .
$$

There can be many such rotation matrices but we will be interested only in the geodesic rotation such that

$$
R_{j i}=\exp (i M s)
$$

where $M$ is a linear combination of $S U(3)$ generators and $s$ is the geodesic distance between $Z_{i}$ and $Z_{j}$ as given in Eq. (27).

The procedure we will adopt is to first consider the special case when $Z_{i}=Z_{0}=(0,0,1)^{T}$. In this case, we can find $R_{j 0}$ and the corresponding $M$. Then we extend the result to include the case when $Z_{i}$ is arbitrary.

$$
\text { 1. } Z_{i}=Z_{0} \text { case: }
$$

Now

$$
Z_{0}^{T}=(0,0,1)
$$

Let us denote

$$
Z_{j}^{T}=\left(z_{1}, z_{2}, z_{3}\right),
$$

where $z_{1}, z_{2}, z_{3}$ are complex numbers and we assume $Z_{j}^{\dagger} Z_{j}=1$.

We wish a matrix $M$ such that

$$
Z_{j}=\exp (i M s) Z_{0} .
$$

The matrix $M$ is a linear combination of $S U(3)$ generators. However, the generators of the unbroken $S U(2) \times$ $U(1)$ sub-group need not be included since they have no effect on $Z_{0}$. So we need only consider $M$ of the form

$$
M=\left(\begin{array}{ccc}
0 & 0 & -i v \\
0 & 0 & -i w \\
i v^{*} & i w^{*} & 0
\end{array}\right),
$$


where $v, w$ are complex numbers. $M$ is normalized using $\operatorname{tr}\left(M^{2}\right)=2$ and so $|v|^{2}+|w|^{2}=1$.

We want to find $v, w$ in terms of $z_{1}, z_{2}, z_{3}$. By the standard procedure of diagonalizing $M$ or by using the formula $M^{3}=M$, one finds

$$
\begin{aligned}
& R_{j 0}=e^{i M s} \\
& =\left(\begin{array}{ccc}
|v|^{2} \cos s+|w|^{2} & -v w^{*}(1-\cos s) & v \sin s \\
-v^{*} w(1-\cos s) & |v|^{2}+|w|^{2} \cos s & w \sin s \\
-v^{*} \sin s & -w^{*} \sin s & \cos s
\end{array}\right)
\end{aligned}
$$

Now we can relate $v, w$ to $z_{1}, z_{2}, z_{3}$. We have

$$
Z_{j}=\left(\begin{array}{c}
z_{1} \\
z_{2} \\
z_{3}
\end{array}\right)=R_{j 0} Z_{0}=\left(\begin{array}{c}
v \sin s \\
w \sin s \\
\cos s
\end{array}\right) .
$$

and so, in terms of the parametrization (7),

$$
s=\bar{\theta}, \quad v=\cos \bar{\phi} e^{i \alpha}, \quad w=\sin \bar{\phi} e^{i \beta} .
$$

Note that, from Eq. (27), the distance between $Z_{0}$ and $Z_{j}$ is $s$. This shows that the matrix $\exp (i M s)$ is indeed the $S U(3)$ transformation (labeled by $s$ ) that traces a geodesic from $Z_{0}$ to $Z_{j}$. Note also that because in our convention (7) the third component of $Z_{j}$ is real, there is no need for an extra phase factor here.

It can also be verified by explicit substitution that one may write $R_{j 0}$ in terms of $Z_{0}$ and $Z_{j}$ as

$$
R_{j 0}=\mathbf{1}-\frac{\left(Z_{0}+Z_{j}\right)\left(Z_{0}^{\dagger}+Z_{j}^{\dagger}\right)}{1+Z_{j}^{\dagger} Z_{0}}+2 Z_{j} Z_{0}^{\dagger} .
$$

Next, we relax the condition $Z_{i}=Z_{0}$.

\section{General $Z_{i}$ case:}

We would like to find $R_{j i}$ such that

$$
R_{j i} Z_{i} \cong Z_{j}
$$

where $R_{j i}=\exp (i M s)$ and $s$ is the geodesic distance between arbitrary points $Z_{i}$ and $Z_{j}$ in $\mathbb{C} P^{2}$.

We already know how to construct the matrix $R_{i 0}$ as in Eq. (B7) that rotates from $Z_{0}$ to $Z_{i}$. Next find the point

$$
Z_{\bar{j}}=R_{i 0}^{\dagger} Z_{j}
$$

where the bar on the subscript $j$ in $Z_{\bar{j}}$ denotes that the point is obtained by rotating $Z_{j}$. It is important to note that the third component of $Z_{\bar{j}}$ may not be real. In fact, since scalar products are unchanged by $S U(3)$ transformations, the third components is $Z_{0}^{\dagger} Z_{\bar{j}}=Z_{i}^{\dagger} Z_{j}$.

Next we find $R_{\bar{j} 0}$ such that

$$
R_{\bar{j} 0} Z_{0} \cong Z_{\bar{j}}
$$

where to use the result in Eq. (B7) or (B10) requires removing the phase factor, i.e.,

$$
R_{\bar{j} 0} Z_{0}=Z_{\bar{j}} \frac{Z_{j}^{\dagger} Z_{i}}{\left|Z_{j}^{\dagger} Z_{i}\right|}
$$

Then it is straightforward to check that

$$
R_{j i} Z_{i}=Z_{j} \frac{Z_{j}^{\dagger} Z_{i}}{\left|Z_{j}^{\dagger} Z_{i}\right|} \cong Z_{j}
$$

where

$$
R_{j i}=R_{i 0} R_{\overline{j 0}} R_{i 0}^{\dagger}
$$

Note that the rotation $R_{j i}$ is the shortest such rotation since $R_{\bar{j} 0}$ is the shortest rotation from $Z_{0}$ to $Z_{\bar{j}}$. The $R_{i 0}$ transformations in Eq. (B16) translate the geodesic path from $Z_{0}$ to $Z_{\bar{j}}$ such that it now goes from $Z_{i}$ to $Z_{j}$.

It is also possible to write an explicit formula analogous to (B10) for $R_{j i}$. In fact, we have simply to replace $Z_{0}$ in that formula by $Z_{i}$ and $Z_{j}$ by $Z_{j}\left(Z_{j}^{\dagger} Z_{i} /\left|Z_{j}^{\dagger} Z_{i}\right|\right)$.

\section{APPENDIX C: CONSTRUCTION OF THE MATRIX $S$.}

The matrix $S_{j i}$ is an $S U(2)$ geodesic rotation that tranforms $\left(A_{j i}, B_{j i}\right)$ to $\left(A_{j}, B_{j}\right)$ at the point $Z_{j}$ on $\mathbb{C} P^{2}$ (see Fig. 3 and Eq. (31)). These are the well-known Euler rotations e.g. see Section 4.5 in 21].

First we apply the rotations $R_{j 0}^{\dagger}$ to parallel transport all quantities from $Z_{j}$ to $Z_{0}$ where we know that the unbroken $S U(2)$ lies in the $1-2$ block of the generators. Quantities at $Z_{0}$ will carry a (0) superscript e.g. $\left(A_{j i}^{(0)}, B_{j i}^{(0)}\right)$ and $\left(A_{j}^{(0)}, B_{j}^{(0)}\right)$. Then we perform an $S U(2)$ rotation $S_{j i}^{(0)}$ that rotates $\left(A_{j i}^{(0)}, B_{j i}^{(0)}\right)$ to $\left(A_{j}^{(0)}, B_{j}^{(0)}\right)$. There are two such rotations, each of which can be written as

$$
S_{j i}^{(0)}=\left(\begin{array}{cc}
e^{i \mathbf{n} \cdot \boldsymbol{\sigma} \Phi / 2} & 0 \\
0 & 1
\end{array}\right)
$$

where $\boldsymbol{\sigma}$ denotes the three Pauli spin matrices, and $\psi, \theta$ and $\phi$ are the Euler angles. The angle of rotation, $\Phi$, is given up to a two-fold ambiguity,

$$
\cos \frac{\Phi}{2} \equiv \pm \cos \frac{\phi+\psi}{2} \cos \frac{\theta}{2}
$$

and

$$
\mathbf{n}=\frac{\mathbf{e}}{\sin (\Phi / 2)}
$$

with

$$
\begin{aligned}
& e_{1}=\cos \frac{\phi-\psi}{2} \sin \frac{\theta}{2}, \\
& e_{2}=\sin \frac{\phi-\psi}{2} \sin \frac{\theta}{2}, \\
& e_{3}=\sin \frac{\phi+\psi}{2} \cos \frac{\theta}{2} .
\end{aligned}
$$


The Euler angles $\phi, \psi$ and $\theta$ can be written in terms of the vector triads at $Z_{0},\left(\mathbf{a}_{j i}^{(0)}, \mathbf{b}_{j i}^{(0)}, \mathbf{c}_{j i}^{(0)}\right)$ and $\left(\mathbf{a}_{j}^{(0)}, \mathbf{b}_{j}^{(0)}, \mathbf{c}_{j}^{(0)}\right)$ where $\mathbf{c}=\mathbf{a} \times \mathbf{b}$ :

$$
\begin{aligned}
\cos \theta & =\mathbf{c}_{i j}^{(0)} \cdot \mathbf{c}_{j}^{(0)}, \\
\cos \psi & =\mathbf{a}_{j}^{(0)} \cdot \boldsymbol{\zeta}, \\
\sin \psi & =\left(\mathbf{a}_{j}^{(0)} \times \boldsymbol{\zeta}\right) \cdot \mathbf{c}_{j}^{(0)}, \\
\cos \phi & =\mathbf{a}_{j i}^{(0)} \cdot \boldsymbol{\zeta}, \\
\sin \phi & =\left(\mathbf{a}_{j i}^{(0)} \times \boldsymbol{\zeta}\right) \cdot \mathbf{c}_{j i}^{(0)},
\end{aligned}
$$

where $\boldsymbol{\zeta}$ is a unit vector along the "line of nodes"

$$
\zeta \equiv \frac{\mathbf{c}_{j i}^{(0)} \times \mathbf{c}_{j}^{(0)}}{\left|\mathbf{c}_{j i}^{(0)} \times \mathbf{c}_{j}^{(0)}\right|} .
$$

Finally, the matrix $S_{j i}^{(0)}$ can be parallel transported back to $Z_{j}$ to obtain

$$
S_{j i}=R_{j 0} S_{j i}^{(0)} R_{j 0}^{\dagger} .
$$

The two-fold ambiguity in the rotation corresponds to two possible angles of rotation, by $\Phi$ or by $\Phi-2 \pi$. We choose the rotation that is smaller i.e. $|\Phi| \leq \pi$.

\section{APPENDIX D: CONSISTENCY OF MONOPOLE AND STRING NUMBERS}

The topology of the symmetry breaking scheme described by Eqs. (11) followed by (2) requires that a cell with a nonzero monopole number has an odd number of strings through its faces, while one with zero charge has an even number. Here we demonstrate that the formalism described above respects this condition.

For this purpose it is convenient to rotate all the relevant quantities to the base point $Z_{0}$. In particular, we consider, in place of (35) the quantity

$$
\begin{aligned}
W_{\{i j k\}}^{(0)} & =R_{i 0}^{\dagger} W_{\{i j k\}} R_{i 0} \\
& =S_{\{i j k\}}^{(0)} \exp \left(\frac{1}{2} i \alpha_{\{i j k\}} \sqrt{3} T_{0}^{8}\right),
\end{aligned}
$$

where

$$
S_{\{i j k\}}^{(0)}=R_{i 0}^{\dagger} S_{\{i j k\}} R_{i 0} .
$$

Clearly, $W_{\{i j k\}}^{(0)}$ must be one of the two central elements of $S U(2)_{0}$, and consequently $S_{\{i j k\}}^{(0)} \in U(1)_{0}$ since the other two factors in (D1) are in that subgroup.

Now consider the product of the $W^{(0)}$ s from all four faces, say

$$
W^{(0)}=W_{\{123\}}^{(0)} W_{\{142\}}^{(0)} W_{\{134\}}^{(0)} W_{\{243\}}^{(0)} .
$$

The order of the four factors is arbitrary but has been chosen for later convenience. This product is evidently again one of the two central elements of $S U(2)_{0}$; which one determines whether the number of strings entering the cell is even or odd.

Since $T_{0}^{8}$ commutes with all the $S_{\{i j k\}}^{(0)}$, when we substitute from (D1) into (D3), we can move all the exponential factors to the right, and so write $W^{(0)}$ as a product

$$
W^{(0)}=S^{(0)} \exp \left(i \pi Q \sqrt{3} T_{0}^{8}\right),
$$

where we have used Eq. (21), and

$$
S^{(0)}=S_{\{123\}}^{(0)} S_{\{142\}}^{(0)} S_{\{134\}}^{(0)} S_{\{243\}}^{(0)} .
$$

Moreover, using Eq. (32), we see that each factor here may be written as a product of three factors coming from the edges of the triangle, each transported to $Z_{0}$ :

$$
S_{\{i j k\}}^{(0)}=U_{i k}^{(0)} U_{k j}^{(0)} U_{j i}^{(0)},
$$

where, for example,

$$
U_{j i}^{(0)}=R_{j 0}^{\dagger} S_{j i} R_{j i} R_{i 0} .
$$

The key now is to compare the transformations $U_{j i}^{(0)}$ and $U_{i j}^{(0)}$. By construction, $S_{j i} R_{j i}$ transforms $\Phi_{i}, \Psi_{1 i}$, $\Psi_{2 i}$ into $\Phi_{j}, \Psi_{1 j}, \Psi_{2 j}$, whereas $S_{i j} R_{i j}$ performs the inverse transformation. Moreover, the prescription for choosing between the two possible transformations is the same in each case. These two products are therefore inversses. Thus we learn that

$$
U_{j i}^{(0) \dagger}=U_{i j}^{(0)} .
$$

Now when we substitute (D6) into (D5) we find

$$
\begin{aligned}
S^{(0)}= & U_{13}^{(0)} U_{32}^{(0)} U_{21}^{(0)} \cdot U_{12}^{(0)} U_{24}^{(0)} U_{41}^{(0)} \\
& \times U_{14}^{(0)} U_{43}^{(0)} U_{31}^{(0)} \cdot U_{23}^{(0)} U_{34}^{(0)} U_{42}^{(0)} .
\end{aligned}
$$

These factors are six pairs of mutual inverses, although since they do not necessarily commute, it is not immediately obvious that they cancel. It is clear, however, that two pairs cancel at once, leaving us with

$$
S^{(0)}=U_{13}^{(0)} U_{32}^{(0)} \cdot U_{24}^{(0)} \cdot U_{43}^{(0)} U_{31}^{(0)} \cdot U_{23}^{(0)} U_{34}^{(0)} U_{42}^{(0)} .
$$

But now recall that the product of the last three factors is $S_{\{243\}}^{(0)} \in U(1)_{0}$. Consequently, this product commutes with all the $U^{(0)} \mathrm{s}$, so we may move these three factors together to any desired position in the product. Placing them after the first two we find

$$
S^{(0)}=U_{13}^{(0)} U_{32}^{(0)} \cdot U_{23}^{(0)} U_{34}^{(0)} U_{42}^{(0)} \cdot U_{24}^{(0)} \cdot U_{43}^{(0)} U_{31}^{(0)} .
$$

But now it is clear that we can cancel these pairs successively, so that finally we obtain

$$
S^{(0)}=\mathbf{1}
$$


So this factor may be cancelled from the right side of Eq. (D4), which then becomes

$$
W^{(0)}=\exp \left(i \pi Q \sqrt{3} T_{0}^{8}\right),
$$

This shows, as required, that the number of strings is odd or even according as $Q=1$ or 0 .

\section{APPENDIX E: $S U(2)$ MONOPOLES AND STRINGS}

Here we discuss monopoles connected by strings in the model

$$
S U(2) \rightarrow U(1) \rightarrow 1 .
$$

The first symmetry breaking is achieved by giving a VEV to an $S U(2)$ adjoint, equivalent to choosing a unit 3vector (call it $\mathbf{v}$ ). The vacuum manifold is $S U(2) / U(1) \cong$ $S^{2}$. The second symmetry breaking is achieved by giving a VEV to a second $S U(2)$ adjoint, call it a, which is orthogonal to $\mathbf{v}$. At this stage the vacuum manifold is $S^{1}$. Therefore monopoles are formed in the first symmetry breaking and these get connected by strings in the second symmetry breaking.

To simulate monopole formation, we assign unit vectors $\mathbf{v}$, equivalently points on $S^{2}$, to the points on our spatial lattice [19, 20]. A tetrahedral cell gets mapped to a tetrahedron in $S^{2}$ and some of these mappings will be incontractable, implying the existence of a monopole within the tetrahedral cell.

The formation of strings that connect the monopoles is more involved but easy to picture, as in Fig. 8 Since a is orthogonal to $\mathbf{v}$, we can view it as picking a direction on the tangent plane of the $S^{2}$. To determine if there is a string passing through a triangular plaquette of the spatial lattice, we have to parallel transport a between the vertices of the triangle using rotations $R$ and then rotate the transported vectors at the vertices using $S$. This is explained in Fig. 8. The scheme for $S U(3)$ is just a generalization of the scheme for the $S U(2)$ model. The complications are technical in that, instead of the tangent plane, our "vectors" at every vertex lie on an $S^{3} / \mathbb{Z}_{2}$ fiber and the geodesics and rotations are harder to determine in practice.
[1] T. W. B. Kibble, J. Phys. A 9, 1387 (1976).

[2] T. Vachaspati and A. Vilenkin, Phys. Rev. D 30, 2036 (1984).

[3] I. Chuang, R. Durrer, N. Turok and B. Yurke, Science 251, 1336 (1991).

[4] M. J. Bowick, et al., Science 263, 943 (1994) arXiv:hep-ph/9208233.

[5] S. Digal, R. Ray and A. M. Srivastava, Phys. Rev. Lett. 83 (1999) 5030 arXiv:hep-ph/9805502.

[6] C. Bäuerle et al., Nature 382, 332 (1996).

[7] V. M. H. Ruutu et al., Nature 382, 334 (1996) arXiv:cond-mat/9512117.

[8] A. Maniv, E. Polturak and G. Koren, Phys. Rev. Lett. 91, 197001 (2003) arXiv:cond-mat/0304359.

[9] R. Monaco, M. Aaroe, J. Mygind, R.J. Rivers and V.P. Koshelets, Phys. Rev. B 74, 144513 (2006) arXiv:cond-mat/0607021.

[10] T. W. B. Kibble, G. Lazarides and Q. Shafi, Phys. Lett. B 113 (1982) 237.

[11] M. Daniel, G. Lazarides and Q. Shafi, Nucl. Phys. B 170,
156 (1980).

[12] A. D. Linde, Phys. Lett. B 96, 293 (1980).

[13] P. Langacker and S. Y. Pi, Phys. Rev. Lett. 45, 1 (1980).

[14] I. Bengtsson, J. Braennlund and K. Zyczkowski, Int. J. Mod. Phys. A 17, 4675 (2002) arXiv:quant-ph/0108064.

[15] M. Aryal, A. E. Everett, A. Vilenkin and T. Vachaspati, Phys. Rev. D 34, 434 (1986).

[16] T. Vachaspati and A. Vilenkin, Phys. Rev. D 35, 1131 (1987).

[17] M. Hindmarsh and P. M. Saffin, JHEP 0608, 066 (2006) arXiv:hep-th/0605014.

[18] E. J. Copeland and P. M. Saffin, JHEP 0511, 023 (2005) arXiv:hep-th/0505110.

[19] E. J. Copeland, D. Haws, T. W. B. Kibble, D. Mitchell and N. Turok, Nucl. Phys. B 298, 445 (1988).

[20] R. Leese and T. Prokopec, Phys. Lett. B 260, 27 (1991).

[21] "Classical Mechanics" (second edition), H. Goldstein (1980). 


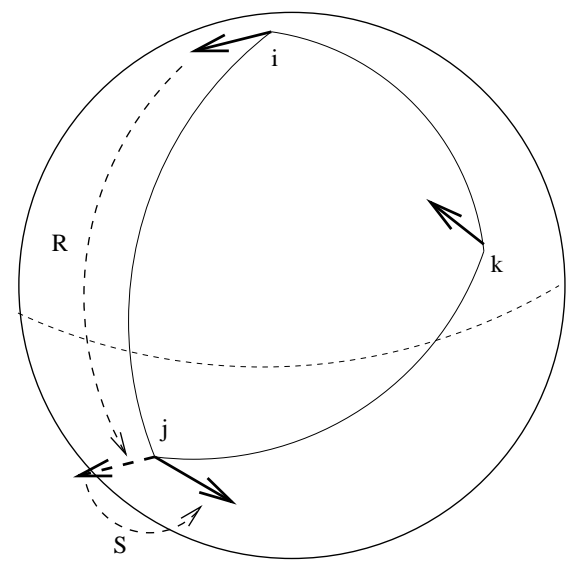

FIG. 8: To determine if a string passes through a spatial triangular plaquette, we first take the corresponding triangle on $S^{2}$, labelled $\{i j k\}$, and then determine if the vector in the tangent plane rotates by $2 \pi$ in circumnavigating the spherical triangle. To do this, we first parallel transport the vector from $i$ to $j$ along a geodesic, described here as a rotation, $R$. Then we find the rotation $S$ within the tangent plane that takes the transported vector into the vector at the vertex $j$. In each case we choose the minimal-angle rotation. Then we do the same thing for the remaining sides. Since we end up with the same vector at $i$ that we started with, the combined transformation is either the identity or a $2 \pi$ rotation. 